\title{
Synthesis, Surfactant Properties and Antimicrobial Activities of Methyl Glycopyranoside Ethers
}

Dorine Belmessieri, ${ }^{[a, c, d]}$ Charlotte Gozlan, ${ }^{[a, e]}$ Marie-Christine Duclos, ${ }^{[a]}$ Valérie Molinier, ${ }^{[b]}$ Jean-Marie Aubry, $^{[b]}$ Oana Dumitrescu, ${ }^{[c, d]}$ Gérard Lina, ${ }^{[c, d]}$ Andreas Redl, ${ }^{[e]}$ Nicolas Duguet, ${ }^{[a]}$ and Marc Lemaire*[a]

Keywords: Antimicrobial • Carbohydrate • Ether • Gram-positive bacteria • Surfactant

[a] $\mathrm{Dr}$ D. Belmessieri, C. Gozlan, M.-C. Duclos, Dr N. Duguet, Prof. M. Lemaire Univ Lyon, Université Claude Bernard Lyon1, CNRS, INSA-Lyon, CPE- Lyon, Institut de Chimie et Biochimie Moléculaires et Supramoléculaires, (ICBMS), UMR 5246, Equipe CAtalyse, SYnthèse et ENvironnement (CASYEN), 43 boulevard du 11 novembre 1918, F-69622 Villeurbanne Cedex, France. E-mails: nicolas.duguet@univ-lyon1.fr , marc.lemaire.chimie@univ-lyon1.fr

[b] Dr V. Molinier, Prof. J.-M. Aubry

Univ. Lille, CNRS, ENSCL, UMR 8181 - UCCS - Unité de Catalyse et Chimie du Solide F-59000 Lille, Cité Scientifique, France.

[c] Dr D. Belmessieri, Dr O. Dumitrescu, Prof. G. Lina Univ Lyon, Université Claude Bernard Lyon 1, Ecole Normale Supérieure de Lyon, CNRS, UMR5308, International Center for Infectiology Research (CIRI), Inserm U1111, 7 rue Guillaume Paradin, F-69008 Lyon, France

[d] Dr D. Belmessieri, Dr O. Dumitrescu, Prof. G. Lina Centre National de Référence des Staphylocoques 59 boulevard Louis Pinel, F-69677 Bron Cedex, France

[e] Dr A. Redl

Tereos Syral SAS, Z.I et Portuaire, B.P.32, 67390 Marckolsheim, France. 
Abstract: A series of amphiphilic methyl glucopyranoside ethers incorporating various alkyl chain lengths has been synthesized from commercially available methyl glucopyranosides following an acetalization/hydrogenolysis sequence. The amphiphilic properties of ethers and acetal intermediates were evaluated. Both families exhibit excellent surfactant properties with a maximum efficiency obtained for compounds bearing a linear dodecyl chain $\left(C M C=0.012 \mathrm{mM}, \gamma_{\text {sat. }}=30 \mathrm{mN} . \mathrm{m}^{-}\right.$ $\left.{ }^{1}\right)$. Antimicrobial activity studies revealed an efficient activity $(0.03<$ MIC $<0.12 \mathrm{mM})$ against Grampositive bacteria such as Listeria monocytogenes, Enterococcus faecalis, Enterococcus faecium and Staphylococcus aureus. More importantly, these compounds were found to be active against multiresistant strains such as vancomycin-, methicillin- and daptomycin-resistant strains. Finally, it was found that antimicrobial activities are closely related to physicochemical properties and are also influenced by the nature of the carbohydrate moiety.

\section{Introduction}

Antibiotics and other antimicrobials are essential compounds to inhibit or prevent the growth of undesirable microorganisms. ${ }^{[1]}$ In this respect, they are widely used to prevent and cure bacterial infection and they have found many applications in medicine, in food industry, ${ }^{[2]}$ and in other fields such as packaging ${ }^{[3]}$ and textile. ${ }^{[4]}$ However, the consequence of this extensive utilization is the emergence of antimicrobial-resistant infectious agents, which represents a major problem for public health. ${ }^{[5]}$ It is becoming particularly alarming since all known antimicrobial chemotherapies are striving to cure superbug's infections. ${ }^{[6]}$ That is the reason why there is an incentive to seek and develop new effective agents with improved activity against resistant pathogens. ${ }^{[7]}$ Moreover, the attention should also be paid on the cost of such agents in order to guarantee their accessibility to a large part of the World's population. Where is the progress if we have in hands highly sophisticated antimicrobials that no one can afford?

In this context, cheap antimicrobials based on long-chain fatty acids and their corresponding polyol ester derivatives have been developed. One of the most active is monolaurin, a linear C12 glycerol monoester, manufactured under the trademark Lauricidin ${ }^{\circ}$. This compound is used as food additive in order to inhibit the growth of various microorganisms. ${ }^{[8]}$ Carbohydrate-derived antimicrobials have also attracted a lot of attention. Indeed, carbohydrates are cheap, natural products, available in bulk quantities from renewable resources. Moreover, they are usually non-toxic and bio-degradable. As a result, they provide an adequate platform for antimicrobials targeting industrial applications. For example, sucrose esters are common carbohydrate fatty acid esters used by the food industry as preservatives. ${ }^{[9]}$ Particularly, sucrose monolaurate was found to be active against Listeria 
monocytogenes and, to a lesser extent, against Staphylococcus aureus. ${ }^{[10]}$ Other carbohydrate fatty acid esters, such as 6'-O-lauroylmaltose and 6"-O-lauroylmaltotriose were found to inhibit the growth of Streptococcus sobrinus, hence they are promising additives in oral-hygiene products. ${ }^{[1]}$ Moreover, these molecules revealed to be active against Bacillus spp. and Lactobacillus plantarum. ${ }^{[12]}$ Monosaccharide fatty acid esters have also shown interesting antimicrobials properties. For example, methyl 6-O-lauroyl- $\alpha$-D-glucopyranoside displayed activity against S. aureus and Escherichia coli. ${ }^{[13]}$ Some other analogues were also evaluated and found to be active against $L$. innocua and $L$. monocytogenes. ${ }^{[14]}$ From a mechanistic point of view, carbohydrate fatty acid esters exhibit antimicrobial activity due to their ability to interact with biological membranes. ${ }^{[15]}$ Indeed, their surfactant properties allow the solubilisation of the lipids constituting the cell membrane. This phenomenon impairs the membrane integrity and causes cellular lysis. ${ }^{[16]}$ However, the ester bond of these antimicrobials could be cleaved by cellular esterases, thus releasing the corresponding inactive sugar and free fatty acid. ${ }^{[13 b]}$ For example, Ruzin and Novick have shown that monolaurin is rapidly hydrolyzed ( $t_{1 / 2}$ of about $5 \mathrm{~min}$ ) in the presence of $S$. aureus cells. ${ }^{[17]}$

To prevent the hydrolysis of these antimicrobial agents, the ester linkage can be advantageously replaced by an ether function, insensitive to esterases. For example, the corresponding ether of monolaurin, 1-O-dodecylglycerol, has shown greater activity against E. faecium than monolaurin itself, probably due to its greater retention in the cell. ${ }^{[18]}$ Similar observations were made with carbohydrate fatty ethers such as methyl 6-O-dodecanyl- $\alpha$-D-glucopyranoside, which exhibits an enhanced activity against $S$. aureus ${ }^{[13]}$ and Listeria spp. in comparison to its corresponding ester. ${ }^{[14]}$ Consequently, carbohydrate fatty ethers seem to be promising candidates for new antimicrobial agents with improved activity and half-life in cells. However, the synthesis of carbohydrate-derived ethers usually requires a multi-step route including protection/deprotection strategies for selectivity and solubility reasons. In addition, the ether function is usually introduced using Williamson conditions requiring a strong base and an alkyl halide or pseudo-halide. ${ }^{[19]}$ As a result, the atom economy of such routes is usually quite low and large amount of waste is produced. Finally, such strategies result in a very high production cost which is not suitable for widespread industrial applications. This could probably explain why, to date, no carbohydrate fatty ether antimicrobial is available on the market. In this context, our group has recently developed an efficient methodology to prepare alkyl ethers of carbohydrate derivatives through hydrogenolysis of the corresponding acetals. ${ }^{[20]}$ Herein, we report our efforts to improve and extend the scope of application of this methodology to a range of methyl glycopyranosides. We also describe the amphiphilic properties and antimicrobial activities of a series of methyl glycopyranoside ethers and their acetal intermediates. ${ }^{[21]}$ 


\section{Results and Discussion}

\section{Synthesis of methyl $\alpha$-D-glucopyranoside alkyl ethers}

The Dunne's group described the synthesis of methyl 6-O-dodecyl- $\alpha$-D-glucopyranoside from commercially available methyl $\alpha$-D-glucopyranoside 1 following traditional carbohydrate chemistry (Scheme 1, a). ${ }^{[13 a]}$

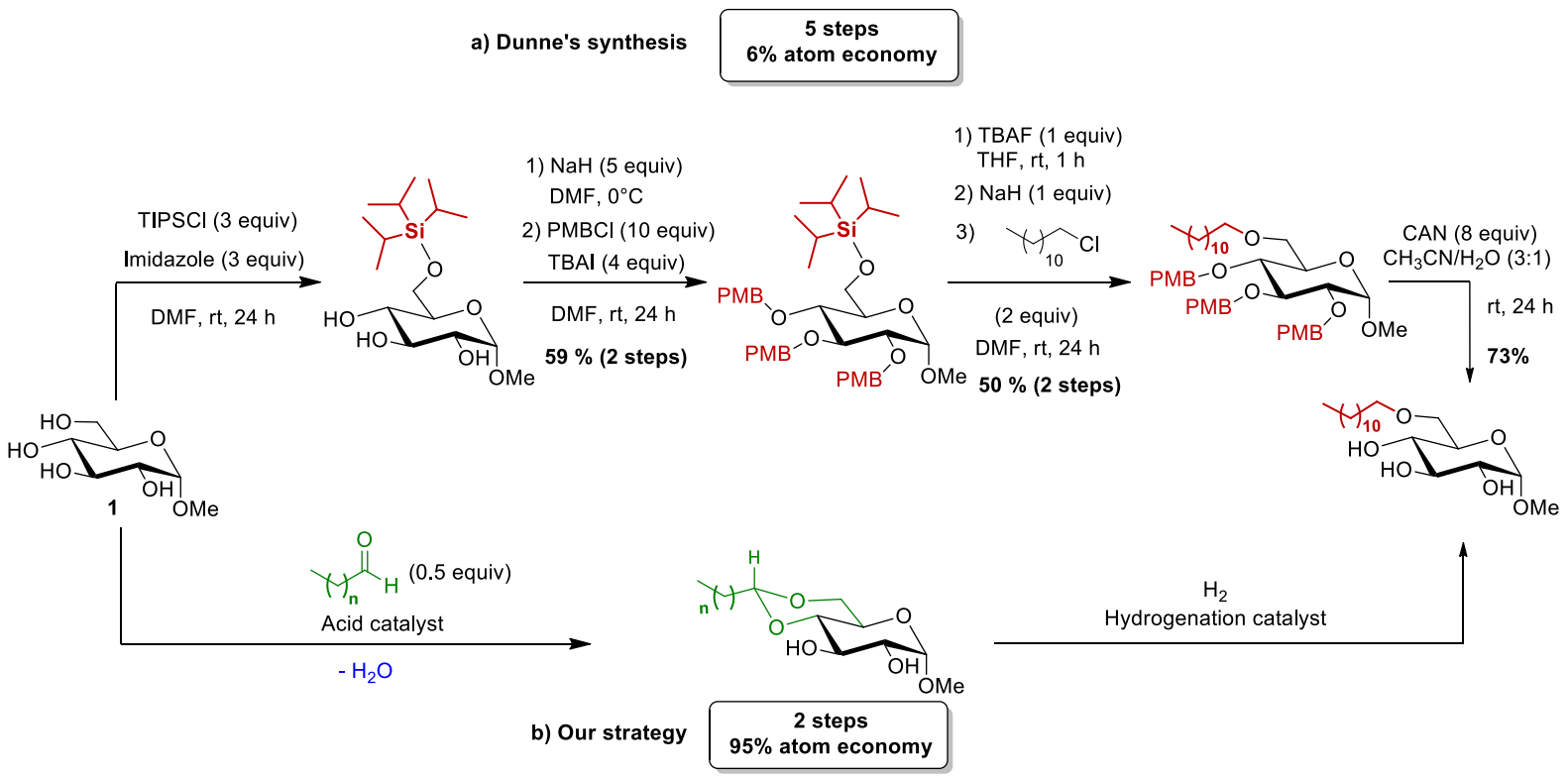

Scheme 1: Comparison of synthetic routes to access methyl 6-O-dodecyl- $\alpha$-D-glucopyranoside. TIPS = triisopropylsilyl, DMF $=$ dimethylformamide, $\mathrm{PMB}=$ para-methoxybenzyl, $\mathrm{TBAI}=$ tetrabutylammonium iodide, $\mathrm{TBAF}=$ tetrabutylammonium fluoride, CAN = cerium ammonium nitrate.

This five-step sequence involves 13 elemental reactions including TIPS-protection of the primary alcohol, PMB-protection of the secondary alcohols, selective TBAF-deprotection of the primary alcohol followed by the installation of the dodecyl chain using Williamson conditions and final secondary alcohols deprotection with CAN. The desired product was obtained as a single stereoisomer in $21 \%$ overall yield and $6 \%$ atom economy. ${ }^{[22]}$ However, this type of synthetic protocol is no longer in line with the green chemistry principles. ${ }^{[23]}$ Consequently, we have developed a twostep acetalisation / hydrogenolysis sequence for the preparation of methyl $O$-alkyl- $\alpha$-Dglucopyranoside directly from unprotected methyl $\alpha$-D-glucopyranoside 1 (Scheme 1, b). Following our previously reported procedure, ${ }^{[20]}$ a range of methyl $\alpha$-D-glucopyranoside 4,6-O-acetals 2-6 bearing different alkyl chain lengths were synthesized in $26-44 \%$ isolated yields (Scheme 2). Subsequent hydrogenolysis of the acetals afforded the corresponding ethers 7-11 as a mixture of 6$O$ - and $4-O$ - regioisomers in up to $75: 25$ selectivity and in $37-81 \%$ isolated yields (Scheme 2). Methyl 6-O-dodecyl- $\alpha$-D-glucopyranoside $\mathbf{1 1}$ was prepared in $10 \%$ overall yield but with an improved $95 \%$ 
atom economy. ${ }^{[22]}$ Considering the atom efficiency (1.3\% vs $\left.11.2 \%\right)$, this strategy is 8.5 times more efficient than Dunne's synthesis. ${ }^{[22]}$ This cheap and environmentally friendly procedure provides a remarkable improvement in carbohydrate chemistry as it allows a convenient and rapid access to alkylated sugars.

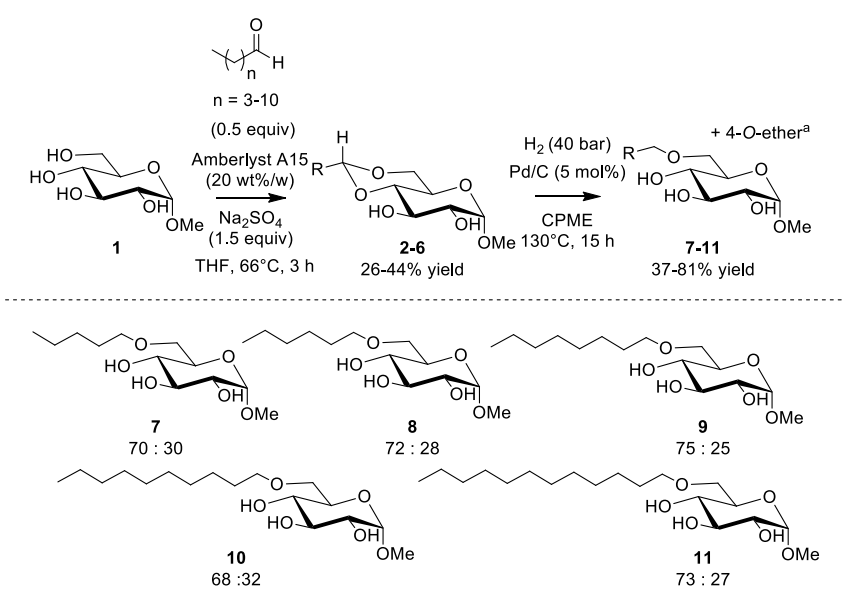

Scheme 2. Preparation of methyl $\alpha$-D-glucopyranoside ethers by acetalisation / hydrogenolysis sequence. ${ }^{a}$ The $6-O: 4-O$ regioisomeric ratio was determined by GC chromatography after derivatization. CPME = cyclopentyl methyl ether.

\section{Amphiphilic properties of methyl $\alpha$-D-glucopyranoside acetals and ethers}

The surface tension of aqueous solutions of acetals 2-6 and ethers 7-11 was evaluated by the rod method, based on the Wilhelmy plate method. The amphiphilic properties of acetals 2-6 (abbreviated CXAcMeGlu, with $\mathrm{x}=$ number of carbons of the alkyl chain, $\mathrm{Ac}=$ acetal, MeGlu = methyl $\alpha$-D-glucopyranoside) were first investigated (Figure 1). All acetals allow a decrease of the surface tension of water to a plateau of about $30 \mathrm{mN} . \mathrm{m}^{-1}$. Different tensiometric curve profiles were observed depending on the alkyl chain length (Figure 1). Neither clear break nor plateau was obtained for C5AcMeGlu 2 and C6AcMeGlu 3, typical of hydrotropes. ${ }^{[24]}$ Indeed, the alkyl chain of these amphiphiles is too short to promote cooperative association into micelles. Nevertheless, when increasing the concentration they accumulate at the water/air interface and form small aggregates within the aqueous solution thus, lowering the superficial tension. Typical surfactant profiles were yet observed for compounds C8AcMeGlu $\mathbf{4}$ and C10AcMeGlu $\mathbf{5}$. The limit of solubility of $\mathbf{4}$ and $\mathbf{5}$ in water revealed to be close to the $\mathrm{CMC}$ value implying a very small concentration range for micellar solubilisation. Expectedly, the increase of the alkyl chain length led to a decrease of the MHC / CMC values, from $82 \mathrm{mM}$ for C5AcMeGlu 2 to $0.15 \mathrm{mM}$ for C10AcMeGlu 5 (Table 1, entries 1-4). Finally, acetal $6(C x=C 12)$ could not be fully analyzed due to its low solubility in water and the formation of 
liquid crystals at low concentrations and at room temperature. However, its $\mathrm{CMC}$ has been estimated to be $>0.06 \mathrm{mM}$ (Table 1, entry 5).

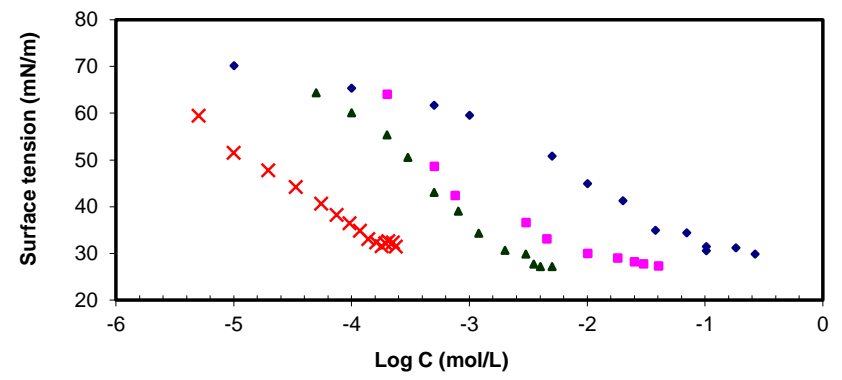

Figure 1. Surface tension vs concentration of methyl $\alpha$-D-glucopyranoside acetals. $\bullet$ C5AcMeGlu 2, $=$ C6AcMeGlu 3, C8AcMeGlu 4, X C10AcMeGlu 5.

Table 1. Minimal Hydrotropic Concentration (MHC), Critical Micelle Concentration (CMC) and surface tension ( $\gamma$ sat) values of methyl a-D-glucopyranoside acetals.

\begin{tabular}{cccc}
\hline Entry & $\begin{array}{c}\text { Methyl } \alpha \text {-D- } \\
\text { glucopyranoside } \\
\text { acetals }\end{array}$ & $\begin{array}{c}\text { MHC or CMC } \\
\left(\mathrm{mmol}^{[a]} \mathrm{L}^{-1}\right)\end{array}$ & $\begin{array}{c}\gamma_{\text {sat }}{ }^{[\mathrm{a}]} \\
\left(\mathrm{mN} . \mathrm{m}^{-1}\right)\end{array}$ \\
\hline 1 & C5AcMeGlu 2 & 82 & 31 \\
2 & C6AcMeGlu 3 & 6.5 & 31 \\
3 & C8AcMeGlu 4 & 2.5 & 28 \\
4 & C10AcMeGlu 5 & 0.15 & 32 \\
5 & C12AcMeGlu 6 & $>0.06$ & $<33$ \\
& & (solubility limit) & \\
\hline
\end{tabular}

${ }^{[a]}$ Determined by tensiometry.

The surface tension of the regioisomeric mixtures of methyl $\alpha$-D-glucopyranoside ethers 7-11 (abbreviated CxEthMeGlu, with $\mathrm{x}=$ number of carbons of the alkyl chain, Eth = ether, MeGlu = methyl $\alpha$-D-glucopyranoside) was next investigated (Figure 2). Similarly, all ethers decrease the surface tension of water to a plateau around $30 \mathrm{mN} \cdot \mathrm{m}^{-1}$. Considering that the 6-O- : 4-O-regioisomeric ratio is similar (around $70: 30$ ) for all methyl $\alpha$-D-glucopyranoside ethers, the alkyl chain length is the only parameter influencing the MHC and CMC values. This behavior is commonly observed for other types of surfactants. ${ }^{[25]}$ As for acetals, ethers 7-8 bearing a C5 and C6 alkyl chain, exhibit a hydrotropic character with MHC values of 35.0 and $13.4 \mathrm{mM}$, respectively (Table 2, entries 1-2). With longer alkyl chain, C8EthMeGlu 9 and C10EthMeGlu 10 show characteristic surfactant profiles but with CMC 
values poorly defined at low concentrations, i.e. 0.43 and $0.28 \mathrm{mM}$ respectively (Table 2, entries 3-4). Gratifyingly, further increase of the alkyl chain length to 12 carbons permits a decrease of the CMC to $0.012 \mathrm{mM}$ (Table 2, entry 5). For a given alkyl chain, the CMC or MHC values of ethers are usually lower than the acetals implying a higher hydrophilicity for the ether family. This trend was previously observed for sorbitan derivatives. ${ }^{[20 b]}$

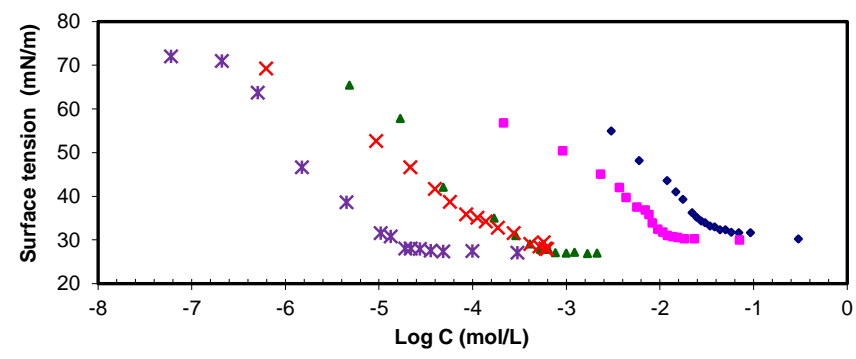

Figure 2. Surface tension vs concentration of methyl $\alpha$-D-glucopyranoside ethers (mixtures of regioisomers). $\bullet$ C5EthMeGlu 7, - C6EthMeGlu 8, $\Delta$ C8EthMeGlu 9, x C10EthMeGlu 10, * C12EthMeGlu 11.

Table 2. Minimal Hydrotropic Concentration (MHC), Critical Micellar Concentration (CMC) and surface tension $\left(\gamma_{\text {sat }}\right)$ values of methyl $\alpha$-D-glucopyranoside ethers (mixtures of regioisomers).

\begin{tabular}{ccccc}
\hline Entry & $\begin{array}{c}\text { Methyl } \alpha \text {-D- } \\
\text { glucopyranoside ethers }\end{array}$ & $\begin{array}{c}\text { Ratio }^{\text {[a] }} \\
(\%)\end{array}$ & $\begin{array}{c}\text { MHC or CMC } \\
\left(\mathrm{mmol}^{[\mathrm{b}]}\right)\end{array}$ & $\begin{array}{c}\gamma_{\text {sat }}{ }^{[\mathrm{b}]} \\
\left(\mathrm{mN} \cdot \mathrm{m}^{-1}\right)\end{array}$ \\
\hline 1 & C5EthMeGlu 7 & $70: 30$ & 35.0 & 33 \\
2 & C6EthMeGlu 8 & $72: 28$ & 13.4 & 31 \\
3 & C8EthMeGlu 9 & $75: 25$ & 0.434 & 30 \\
4 & C10EthMeGlu 10 & $68: 32$ & 0.277 & 28 \\
\hline 5 & C12EthMeGlu 11 & $73: 27$ & 0.012 & \\
\hline
\end{tabular}

[a] 6-O : 4-O regioisomeric ratio. ${ }^{[b]}$ Determined by tensiometry.

To probe the influence of regioisomers on amphiphilic properties, physicochemical analyses were also performed on pure 4-O- and 6-O-C12EthMeGlu 11a and $11 \mathrm{~b}$ regioisomers (Figure 3). Pure regioisomers were separated from the mixture by column chromatography on silica gel. Similar profiles were obtained for both regioisomers, however, 4-O-isomer 11a (brown triangles) showed a short plateau with a solubilisation limit close to its $C M C$, while a better surfactant profile was

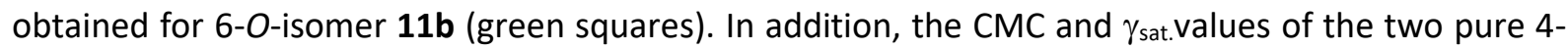
$O$ - and 6-O-isomers are relatively close, with 0.046 and $0.030 \mathrm{mM}$, and 31 and $29 \mathrm{mN} \cdot \mathrm{m}^{-1}$ respectively (Table 3, entries 1-2). Consequently, these results prove that the position of the alkyl chain on the 
sugar scaffold does not induce major changes on neither the surface tension nor the CMC values. Interestingly, when using a $73: 27$ mixture of $6-O-$ and 4-O-isomers 11 (purple crosses), the CMC decreases to $0.012 \mathrm{mM}$ (Table 3, entry 3). This non-ideal behavior is very common when mixing two surfactants. It is attributed to an attractive interaction between two surfactants located at the surface monolayer and within mixed aggregates. This leads to a stabilization of the mixed superficial layer and the co-micelles. ${ }^{[26]}$ We applied the equations of the regular solution theory to this specific isomeric ratio, which gave an interaction parameter $\beta=-4.7$ and a mole fraction of isomer $6-O$ in the mixed micelle $X_{1}=0.61$ (see references $26 \mathrm{a}$ and $26 \mathrm{~b}$ for calculation details). Even if CMC measurements on other isomeric ratios are required to get a more accurate value of interaction parameter, this strong negative $\beta$ value clearly indicates preferential attractive interactions between the two regioisomers.

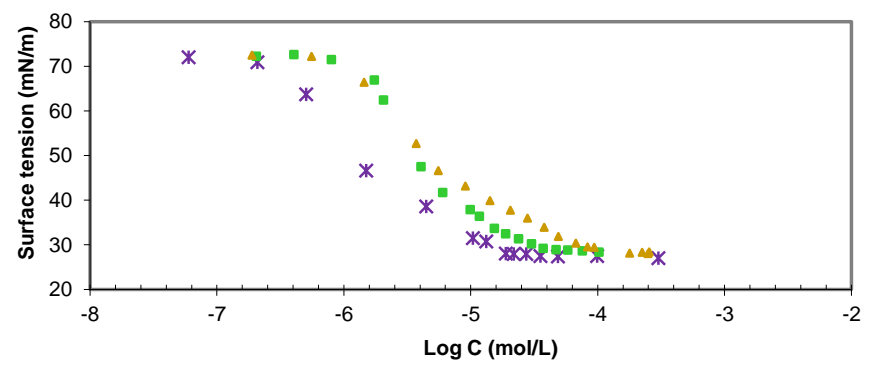

Figure 3. Surface tension vs concentration of methyl dodecyl- $\alpha$-D-glucopyranoside: $\triangle 4$-O-isomer 11a, $\|$ 6-O-isomer 11b, and $*$ a $73: 27$ mixture of 6-O- and 4-O-isomers 11.

Table 3. Critical Micelle Concentration (CMC) and surface tension ( $\left.\gamma_{\text {sat }}\right)$ values of regioisomers 6-O and 4-O of C12EthMeGlu 11.

\begin{tabular}{|c|c|c|c|c|}
\hline Entry & $\begin{array}{l}\text { Methyl } \alpha \text {-D- } \\
\text { glucopyranoside ethers }\end{array}$ & Ratio[a] & $\begin{array}{c}\mathrm{CMC}^{[\mathrm{b}]} \\
\left(\mathrm{mmol} . \mathrm{L}^{-1}\right)\end{array}$ & $\begin{array}{c}\gamma_{\mathrm{sat}}{ }^{[\mathrm{b}]} \\
\left(\mathrm{mN} \cdot \mathrm{m}^{-1}\right)\end{array}$ \\
\hline 1 & $\begin{array}{c}\text { 4-O-C12EthMeGlu } \\
11 a\end{array}$ & $0: 100$ & 0.046 & 31 \\
\hline 2 & $\begin{array}{c}\text { 6-O-C12EthMeGlu } \\
\text { 11b }\end{array}$ & $100: 0$ & 0.030 & 29 \\
\hline 3 & $\begin{array}{c}(6+4)-O-\text { C12EthMeGlu } \\
\mathbf{1 1}\end{array}$ & $73: 27$ & 0.012 & 30 \\
\hline
\end{tabular}

${ }^{[a]}$ 6- $O$ : 4-O regioisomeric ratio. ${ }^{[b]}$ Determined by tensiometry.

In order to evaluate the efficiency of these surfactants, a comparison of methyl 6-O-dodecyl- $\alpha$-Dglucopyranoside 11b with known sugar-based non ionic surfactants was next carried out (Table 4). 
Only compounds bearing a C12 alkyl chain were selected in order to establish a structure / properties relationship. First of all, methyl 6-O-dodecyl- $\alpha$-D-glucopyranoside $(C M C=0.030 \mathrm{mM}$ ) was found by far more efficient than its glucose analog $(C M C=0.5 \mathrm{mM})$, showing the importance of the substitution at the anomeric position (Table 4, entries 1-2). Moreover, the replacement of the ester function in methyl 6-O-dodecanoyl- $\alpha$-D-glucopyranoside $(C M C=0.052 \mathrm{mM})$ by an ether link does not have a significant impact on the CMC (Table 4, entry 3). However, the introduction of a second carbohydrate unit in 6-O-dodecanoyl-sucrose and in 6'-O-dodecanoyl-maltose leads to an important increase of the CMC to 0.25 and $0.24 \mathrm{mM}$, respectively (Table 4, entries 4-5). Higher CMC values were also obtained with the corresponding commercially available dodecyl $\alpha$-D-glucopyranoside and dodecyl maltoside (Table 4, entries 6-7). This means that, in the case of methyl 6-O-dodecyl- $\alpha$-Dglucopyranoside 11, about 12 to 17 times less product is required to obtain the same performances than these commercial surfactants. Finally, this study highlights the potential of ether $\mathbf{1 1}$ as new biosourced non-ionic surfactant with improved robustness in comparison to esters, broadening the scope of applications of such amphiphilic compounds.

Table 4. Comparison of $\mathrm{CMC}$ values (mM) of known sugar-based non-ionic surfactants.

\begin{tabular}{|c|c|c|c|}
\hline Entry & Carbohydrate surfactants & $\mathrm{CMC}\left(\mathrm{mmol} \cdot \mathrm{L}^{-1}\right)$ & Ref. \\
\hline 1 & Methyl 6-O-dodecyl-a-D-glucopyranoside & $\begin{array}{c}0.012 \\
\text { (mixture of } \\
\text { isomers) } \\
0.03 \text { (6-O-isomer) }\end{array}$ & This work \\
\hline 2 & 6-O-Dodecyl-D-glucose & 0.5 & [27] \\
\hline 3 & 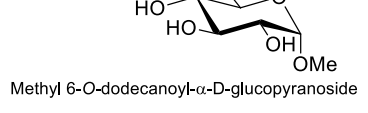 & 0.052 & [28] \\
\hline 4 & 6-O-Dodecanoyl sucrose & 0.25 & [29] \\
\hline 5 & 6'-O-Dodecanoyl-maltose & 0.24 & [29] \\
\hline
\end{tabular}




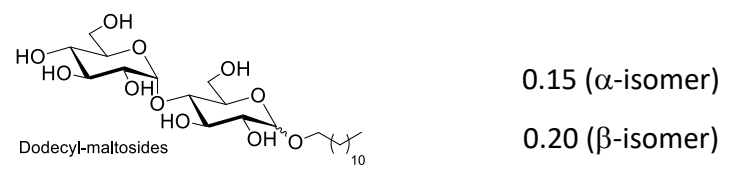

\section{Antimicrobial activities of methyl $\alpha$-D-glucopyranoside acetals and ethers}

The antimicrobial activities of methyl $\alpha$-D-glucopyranoside acetals and ethers were first evaluated against Gram-negative bacteria such as E. coli $\left(\right.$ ATCC $^{\circ} 8739^{\mathrm{TM}}$ ) and Pseudomonas aeruginosa (ATCC ${ }^{\circ}$ $27853^{\mathrm{TM}}$ ) and their efficacy was determined using the Minimum Inhibitory Concentration values (MIC). ${ }^{[32]}$ Unfortunately, no antimicrobial activity was detected against these bacteria at a concentration up to $4.0 \mathrm{mM}$ for neither acetal nor ether derivatives under the conditions used. ${ }^{[33]}$ Similar results were obtained against this type of microorganism in Dunne's studies on carbohydrate fatty esters, with MIC values reaching $10-20 \mathrm{mM} \cdot{ }^{[13 a]}$ The bacterial species tested represent two of the most frequently Gram-negative pathogens isolated in humans, our results suggest that these compounds cannot be used to target Gram-negative bacterial infections. In fact, one of the structural feature of Gram-negative bacteria is the presence of a external membrane surrounding each bacterial cell wall. Thus, one possible explanation would be hypothesized that the derivatives are enable to go through the outer lipopolysaccharide membrane, preventing them to penetrate into the cells.

Consequently, the antimicrobial activities of methyl $\alpha$-D-glucopyranoside acetals and ethers were next evaluated against Gram-positive bacteria, namely, L. monocytogenes (CIP 103575), S. aureus $\left(\right.$ ATCC $^{\circ} 29213^{\mathrm{TM}}$ ) and $E$. faecalis (ATCC ${ }^{\circ} 29212^{\mathrm{TM}}$ ). The results are gathered in Table 5.

Table 5. Antimicrobial activities of methyl $\alpha$-D-glucopyranoside acetals 2-6 and ethers 7-11 against Gram positive bacteria. MICs (Minimum Inhibitory Concentration) in mmol.L-1. MBCs (Minimum Bactericidal Concentration) in mmol. $\mathrm{L}^{-1}$ are given in brackets.

\begin{tabular}{|c|c|c|c|c|}
\hline \multirow[b]{2}{*}{ Entry } & \multirow[b]{2}{*}{ Compounds } & \multicolumn{3}{|c|}{ Bacteria strains } \\
\hline & & $\begin{array}{l}\text { L. mono- } \\
\text { cytogenes } \\
\text { (CIP 103575) }\end{array}$ & $\begin{array}{l}\text { S. aureus } \\
\text { (ATCC }^{\circ} \\
29213^{\mathrm{TM}} \text { ) }\end{array}$ & $\begin{array}{c}\text { E. faecalis } \\
\left(\text { ATCC }^{\oplus} 29212^{\mathrm{TM}}\right)\end{array}$ \\
\hline
\end{tabular}




\begin{tabular}{|c|c|c|c|c|}
\hline 2 & C5EthMeGlu 7 & $>4$ & $>4$ & $>4$ \\
\hline 3 & C6AcMeGlu 3 & $>4$ & $>4$ & $>4$ \\
\hline 4 & C6EthMeGlu 8 & $>4$ & $>4$ & $>4$ \\
\hline 5 & C8AcMeGlu 4 & 2 & $>4$ & $>4$ \\
\hline 6 & C8EthMeGlu 9 & 2 & 2 & $>4$ \\
\hline 7 & C10AcMeGlu 5 & $n d^{[a]}$ & $n d^{[a]}$ & $n d^{[a]}$ \\
\hline 8 & C10EthMeGlu 10 & $0.5(0.5)$ & $2(2)$ & $0.5(0.5)$ \\
\hline 9 & C12AcMeGlu 6 & $\mathrm{nd}^{[\mathrm{a}]}$ & $n d^{[a]}$ & $n d^{[a]}$ \\
\hline 10 & C12EthMeGlu 11 & $0.03(0.03)$ & $0.12(0.12)$ & $0.03(0.03)$ \\
\hline $11^{[b]}$ & C12EthMeGlu 11b & 0.04 & 0.04 & nd \\
\hline $12^{[b]}$ & C12EstMeGlu & 0.08 & 0.32 & nd \\
\hline
\end{tabular}

[a] $\mathrm{Nd}$ : not determined due to the insolubility of the compounds when mixed with the inoculum. [b] MIC values from reference 13

No significant antimicrobial activity ( $\mathrm{MIC}=2$ or $>4 \mathrm{mM}$ ) was detected for acetals and ethers bearing a short C5, C6 or a medium C8 alkyl chain (Table 5, entries 1-6). Furthermore, the MICs of acetals 5 and 6 could not be determined due to their poor solubility in water (Table 5, entries 7 and 9). However, increasing the alkyl chain length of methyl $\alpha$-D-glucopyranoside ethers from C8 to $\mathrm{C} 12$ led to an important decrease of the MIC values (Table 5, entries 6, 8 and 10). The best antimicrobial activities were obtained for C12EthMeGlu 11 (73: 27 mixture of regioisomers) with a MIC of 0.03 $\mathrm{mM}$ for both L. monocytogenes and E. faecalis and of $0.12 \mathrm{mM}$ for the more virulent $S$. aureus strain (Table 5, entry 10). These results are comparable with those obtained with regioisomerically pure methyl 6-O-dodecyl- $\alpha$-D-glucopyranoside 11b (Table 5, entry 11). This reveals that the alkyl chain position has a minor impact on the biological activity. Thus, it highlights the interesting properties of these mixtures obtained through step-economical acetalisation/hydrogenolysis sequence. Finally, ether derivative C12EthMeGlu 11 was found more active than its corresponding ester C12EstMeGlu which is in accordance with the literature ${ }^{[13,18]}$ (Table 5, entry 12).

The bactericidal activities of ethers $\mathbf{1 0}$ and $\mathbf{1 1}$ were then assessed for each bacteria strain by the measurement of the Minimum Bactericidal Concentration (MBC). ${ }^{[34]}$ Indeed, the MBC corresponds to the lowest concentration of antimicrobial agent required to induce the death of a microorganism, 
corresponding to a decrease of the initial bacterial inoculum by at least $99.9 \%$. The MBC values obtained for C10EthMeGlu 10 and C12EthMeGlu 11 were identical to their corresponding MICs close enough to MIC values in order to place them in the same serial dilution interval (Table 5, entries 8 and 10, results in brackets). This observation suggests elearly indicates that these compounds are not only efficient to stop the bacterial growth but also induce the death of the microorganisms.

The antimicrobial activities of methyl $\alpha$-D-glucopyranoside ethers were further compared to their amphiphilic properties (Table 6).

Table 6. Comparison of the Critical Micelle Concentration (CMC) and Minimum Inhibitory Concentration (MIC) of methyl $\alpha$ D-glucopyranoside ethers 7-11.

\begin{tabular}{cccc}
\hline Entry & $\begin{array}{c}\text { Methyl } \alpha \text {-D- } \\
\text { glucopyranoside } \\
\text { ethers }\end{array}$ & $\begin{array}{c}\text { CMC } \\
\left(\mathrm{mmol}^{-1}\right)\end{array}$ & $\begin{array}{c}\text { MIC } \\
\left(\mathrm{mmol}^{-1} \mathrm{~L}^{-1}\right)\end{array}$ \\
\hline 1 & C5EthMeGlu 7 & 35 & $>4$ \\
2 & C6EthMeGlu 8 & 13.4 & $>4$ \\
3 & C8EthMeGlu 9 & 0.434 & $2-4$ \\
4 & C10EthMeGlu 10 & 0.277 & $0.5-2$ \\
5 & C12EthMeGlu 11 & 0.012 & $0.03-0.12$ \\
\hline
\end{tabular}

An increase of the alkyl chain length (from C5 to C12) on methyl $\alpha$-D-glucopyranoside ethers induce both, a decrease of the CMC from 35 to $0.012 \mathrm{mM}$ and of the MIC from $>4$ to about 0.03-0.12 mM. Noticeably, the antimicrobial activities correlate quite well with the amphiphilic properties of compounds 7-11 with MICs about 2 to 10 times the CMC values (Table 6, entries 1-5). These amphiphilic compounds might interact with the cell bi-layer membrane, exerting a variety of effects, ${ }^{[35]}$ thus leading to the cell lysis. Having identified that 12 carbons was the optimal alkyl chain length to reach efficient antimicrobial activity, the effects of the monosaccharide core and the anomeric carbon configuration were next probed. For this purpose, methyl $\alpha$-D-mannopyranoside, $\alpha$ D-galactopyranoside, $\beta$-L-rhamnopyranoside and $\beta$-D-glucopyranoside were subjected to acetalisation with dodecanal to give the corresponding acetals $12-15$ with unoptimized $11-49 \%$ isolated yields. ${ }^{[36]}$ The hydrogenolysis of these acetals gave three new alkylated sugars 16-18 with unoptimized 27-34\% isolated yields $^{[36]}$ (Figure 4). Unfortunately, all attempts to reduce acetal 15 to the desired ether failed under our conditions. This could be explained by the difference of adsorption energies of $\alpha$ - 
and $\beta$-isomers on palladium during the hydrogenolysis step. ${ }^{[37]}$ As a result, only $\beta$-C12AcMeGlu 15 was considered for further tests.

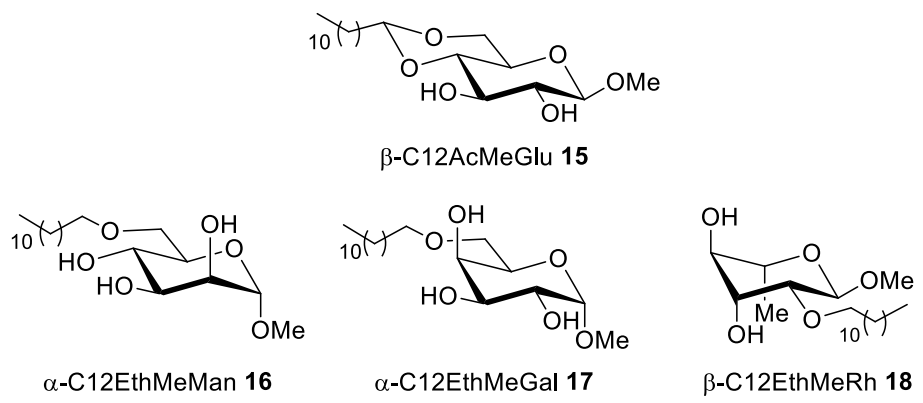

Figure 4. Range of acetal and ether derivatives obtained from acetalisation / hydrogenolysis sequence.

With these molecules in hands, antimicrobial activity studies were next carried out on a wide range of Gram-positive bacteria. The efficacy of the derivatives was first studied against $L$. monocytogenes strains such as the reference (CIP 103575) and three clinical isolated strains (LM1: 015189074801, LM2: 015170199001, LM3 : 015181840701) using amoxicillin as control (Table 7). The glucose derivative 11 gave the best activities against all these strains with MICs of $0.02 \mathrm{mM}$ while mannosebased compound 16 was slightly less active (Table 7, entries 1-2). However, galactose derivative 17 was by far less efficient with a MIC of $0.16 \mathrm{mM}$ on all strains (Table 7, entry 3). Interestingly, rhamnose derivative $\mathbf{1 8}$ was also active but to a lesser extent (Table 7, entry 4). Finally, acetal 15 was also quite active against these L. monocytogenes strains, proving that this class of compounds could also been interesting as antimicrobials, provided their solubility (Table 7, entry 5).

Table 7. Antimicrobial activities of compounds 11, 15-18 against L. monocytogenes strains. MICs (Minimum Inhibitory Concentration) in $\mathrm{mmol} . \mathrm{L}^{-1}$.

\begin{tabular}{cccccc}
\hline \multirow{2}{*}{ Entry } & Compounds & \multicolumn{5}{c}{ L. monocytogenes strains } \\
\cline { 3 - 6 } & & CIP 103575 & LM1 & LM2 & LM3 \\
\hline 1 & C12EthMeGlu 11 & 0.02 & 0.02 & 0.02 & 0.02 \\
2 & C12EthMeMan 16 & 0.09 & 0.02 & 0.04 & 0.04 \\
3 & C12EthMeGal 17 & 0.18 & 0.18 & 0.18 & 0.18 \\
4 & C12EthMeRh 18 & 0.09 & 0.09 & 0.09 & 0.09
\end{tabular}


The activity of compounds $11, \mathbf{1 5 - 1 8}$ was next studied against E. faecalis (ATCC ${ }^{\circ} 29212^{\text {TM }}$ and 015206179901) and E. faecium strains (CIP 103510, Van A 0151850763, 015205731401 and 015205261801) using amoxicillin as control (Table 8). A good level of activity was observed for sugar derivatives 11, 16 and 18 against the six Enterococcus strains studied (MIC $\leq 0.08 \mathrm{mM}$ ), particularly for glucose derivative 11 with a MIC of $0.02 \mathrm{mM}$ for most strains (Table 8, entries 1, 2 and 4). More importantly, this compound was also active against a vancomycin-resistant strain at $0.02 \mathrm{mM}$ (Van A). Once again, galactose derivative $\mathbf{1 7}$ was the least active indicating that the nature of the carbohydrate could affect the antimicrobial efficacy. However, great differences could be observed between strains as the MIC varies from 0.02 to $>0.64 \mathrm{mM}$ (Table 8, entry 3). Similar phenomenon is observed with acetal 15 with MICs ranging from 0.02 to $0.16 \mathrm{mM}$ (Table 8, entry 5)

Table 8. Antimicrobial activities of compounds 11, 15-18 against E. faecalis and E. faecium strains. MICs (Minimum Inhibitory Concentration) in $\mathrm{mmol}^{-\mathrm{L}^{-1}}$.

\begin{tabular}{|c|c|c|c|c|c|c|c|}
\hline \multirow[b]{2}{*}{ Entry } & \multirow[b]{2}{*}{ Compounds } & \multicolumn{2}{|c|}{ E. faecalis strains } & \multicolumn{4}{|c|}{ E. faecium strains } \\
\hline & & ATCC 29212 & 015206179901 & CIP & Van A & $\begin{array}{c}01520573140 \\
1\end{array}$ & 015205261801 \\
\hline 1 & C12EthMeGlu 11 & 0.02 & 0.04 & 0.02 & 0.02 & 0.02 & 0.02 \\
\hline 2 & C12EthMeMan 16 & 0.04 & 0.09 & 0.09 & 0.04 & 0.04 & 0.04 \\
\hline 3 & C12EthMeGal 17 & 0.18 & 0.18 & $>0.71$ & 0.35 & 0.09 & 0.02 \\
\hline 4 & C12EthMeRh $\mathbf{1 8}$ & 0.05 & 0.05 & 0.05 & 0.05 & 0.02 & 0.05 \\
\hline 5 & $\beta$-C12AcMeGlu 15 & 0.18 & 0.09 & 0.09 & 0.09 & 0.04 & 0.02 \\
\hline 6 & amoxicillin & 0.002 & 0.002 & 0.06 & 0.5 & 0.25 & 0.25 \\
\hline
\end{tabular}

The activity of compounds $\mathbf{1 1}, \mathbf{1 5 - 1 8}$ was next studied against a wide range of $S$. aureus strains using oxacillin as control. Two reference strains were considered as well as six methicillin-resistant and three daptomycin-resistant strains (Table 9). Indeed, the development of resistance during therapy is becoming a major health issue highlighted in numerous reports. ${ }^{[38]}$ Similar activities were observed for glucose 11, mannose 16 and rhamnose 18 derivatives with MIC values from 0.04 to $0.08 \mathrm{mM}$ for 
reference strains (Table 9, entries 1, 2 and 4). However, slighly lower activities (0.16-0.32 mM) were observed with acetal 15 (Table 9, entry 5). In accordance with our previous results on Listeria and Enterococcus strains, galactose derivative 17 gave no significant activity with MIC $>0.64 \mathrm{mM}$ for all strains studied (Table 9, entry 4). Gratifyingly, good antimicrobial activities (0.04-0.08 mM) were obtained with glucose derivative $\mathbf{1 1}$ against methicillin- and daptomycin-resistant strains (Table 9, entry 1). Interestingly, a similar level of activity was displayed against sensitive and resistant strains for all compounds.

These results clearly indicate that carbohydrate fatty ethers are promising candidates for the treatment of resistant bacteria. They could find various applications in the food industry, in the medical field and could also be incorporated in various formulations.

Table 9. Antimicrobial activities of compounds 11, 15-18 against S. aureus strains (references), methicillin- and daptomycinresistant S. aureus strains. MICs in mmol. $\mathrm{L}^{-1}$.

\begin{tabular}{|c|c|c|c|c|c|c|c|c|c|c|c|c|}
\hline \multirow[b]{2}{*}{ Entry } & \multirow[b]{2}{*}{ Compounds } & \multicolumn{2}{|c|}{ S. aureus references } & \multicolumn{6}{|c|}{ Methicillin resistant $S$. aureus } & \multicolumn{3}{|c|}{ Daptomycin resistant S. aureus } \\
\hline & & $\begin{array}{l}\text { ATCC } \\
25923\end{array}$ & $\begin{array}{l}\text { ATCC } \\
29213\end{array}$ & $\begin{array}{c}\text { LAC } \\
\text { USA } 300\end{array}$ & MU3 & $\begin{array}{c}\text { HT 2004- } \\
0012\end{array}$ & $\begin{array}{c}\text { LY 199- } \\
0053\end{array}$ & $\begin{array}{c}\text { HT 2002- } \\
0417\end{array}$ & $\begin{array}{c}\text { HT 2006- } \\
1004\end{array}$ & $\begin{array}{c}\text { ST 2015- } \\
0188\end{array}$ & $\begin{array}{c}\text { ST 2014- } \\
1288\end{array}$ & $\begin{array}{c}\text { ST } 2015- \\
0989\end{array}$ \\
\hline 1 & C12EthMeGlu 11 & 0.04 & 0.09 & 0.09 & 0.09 & 0.09 & 0.04 & 0.04 & 0.09 & 0.09 & 0.09 & 0.09 \\
\hline 2 & C12EthMeMan 16 & 0.09 & 0.09 & 0.09 & 0.18 & 0.09 & 0.09 & 0.09 & 0.18 & 0.18 & 0.09 & 0.18 \\
\hline 3 & C12EthMeGal 17 & $>0.71$ & $>0.71$ & $>0.71$ & $>0.71$ & $>0.71$ & $>0.71$ & $>0.71$ & $>0.71$ & $>0.71$ & $>0.71$ & $>0.71$ \\
\hline 4 & C12EthMeRh $\mathbf{1 8}$ & 0.09 & 0.05 & 0.09 & 0.18 & 0.05 & 0.09 & 0.09 & 0.09 & 0.18 & 0.09 & 0.18 \\
\hline 5 & $\beta$-C12AcMeGlu 15 & 0.18 & 0.18 & 0.18 & 0.18 & 0.18 & 0.18 & 0.37 & 0.18 & 0.18 & 0.18 & 0.18 \\
\hline 6 & oxacillin & 0.05 & 0.001 & 0.16 & 0.64 & 0.08 & 0.16 & 0.16 & 0.32 & 0.16 & 0.16 & 0.32 \\
\hline
\end{tabular}

\section{Mechanistic considerations}

We hypothesize that the antimicrobial activity arises from the ability of carbohydrate alkyl ethers to form micelles and thus to solubilize the lipids constituting the cell membrane. However, important MIC differences were observed between C12EthMeGlu 11 and C12EthMeGal 17, indicating that the carbohydrate moiety can also play a crucial role on antimicrobial activity. Consequently, a recognition phenomenon might also be operating between the carbohydrate core and the cell walls of bacteria. Alternatively, modification of the $\mathrm{OH}$ group configuration (from equatorial in glucose to 
axial in galactose) could induce a drastic change of the $\mathrm{CMC}$ also impacting the activity. In order to verify this hypothesis, the CMC value of C12EthMeGal 17 was measured and compared to C12EthMeGlu 11 (Figure 5).

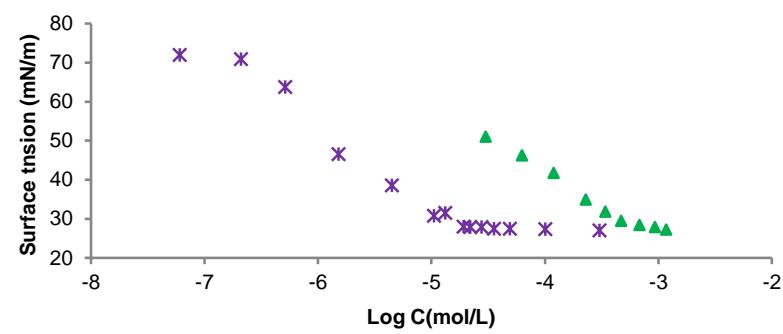

Figure 5. Surface tension of methyl $\alpha$-D-glucopyranoside ether vs methyl $\alpha$-D-galactopyranoside ether $*$ C12EthMeGlu 11, A C12EthMeGal 17.

Table 10. Antimicrobial activities vs CMC of compounds $\mathbf{1 1}$ and $\mathbf{1 7 .}$

\begin{tabular}{cccc}
\hline Entry & Compounds & $\begin{array}{c}\text { MIC }^{[a]} \\
\left(\mathrm{mmol}^{-L^{-1}}\right)\end{array}$ & $\begin{array}{c}\mathrm{CMC}^{[\mathrm{b}]} \\
\left(\mathrm{mmol}^{-1}\right)\end{array}$ \\
\hline 1 & C12EthMeGlu 11 & $0.02-0.08$ & 0.012 \\
2 & C12EthMeGal 17 & $0.02-0.64$ & 0.47 \\
\hline
\end{tabular}

[a] MIC (Minimum Inhibitory Concentration) range including results against against Staphylococcus, Enterrococcus and Listeria strains. ${ }^{[b]}$ Determined by tensiometry.

The $\mathrm{CMC}$ of galactose derivative $\mathbf{1 7}$ is $\mathbf{4 0}$ times higher than glucose $\mathbf{1 1}$ (Table 10). Galactoside $\mathbf{1 7}$ is therefore more hydrophilic than $\mathbf{1 1}$ which is in accordance with previous work on HPLC separation of methyl glycosides. ${ }^{[39]}$ This phenomenon is directly linked to the inversion of the 4-OH group configuration (from equatorial for 11 to axial in 17). Based on Simon's DFT calculations ${ }^{[0]}$ and Hüneberger's conformational analyses, ${ }^{[41]}$ we postulate that the lower hydrophilicity of $\mathbf{1 1}$ is due to the high occurrence of hydrogen-bond networks (Figure 6, left). In the contrary, these interactions are not favored in the case of galactoside $\mathbf{1 7}$ due to the axial 4-OH group, therefore increasing the sugar polarity (Figure 6, right). 


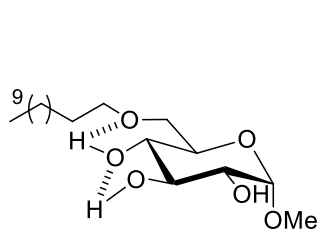

C12EthMeGlu 11

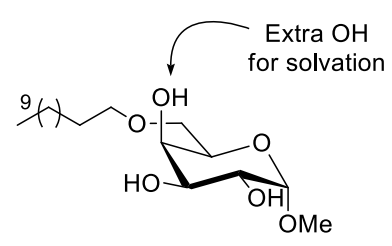

C12EthMeGal 17

Figure 6. Model proposed to explain the polarity difference between C12EthMeGlu 11 and C12EthMeGal 17 (only one conformer showed).

As shown above, minor structural modifications could have significant impacts on the antimicrobial activity. Consequently, we also envisioned to investigate the role of the methyl group in compound 11 by preparing the corresponding demethylated compound. Unfortunately, all attempts to remove the methyl group from compound $\mathbf{1 1}$ following standard conditions (TMS-OTf / $\mathrm{Ac}_{2} \mathrm{O}$ in $\left.\mathrm{CH}_{2} \mathrm{Cl}_{2}\right)^{[42]}$ failed and gave a complex mixture of products. However, based on our previous analysis and the CMC values (Table 4, entries 1-2), we can assume that the antimicrobial activity of 6-O-dodecyl-Dglucose should be considerably lower than the corresponding methyl dodecylglucopyranosides. Further works will be necessary to confirm this hypothesis.

Finally, this study demonstrates that the CMC is the crucial parameter influencing the MIC, however the sugar recognition can still not be ruled out. Further structural/activity studies of sugar-based surfactants and computational analyses are currently on going.

\section{Conclusions}

In conclusion, a series of amphiphilic methyl glycopyranoside alkyl ethers were prepared from the corresponding unprotected methyl glycopyranosides following a two-step acetalization/hydrogenolysis sequence. This route provides a straightforward access to carbohydrate alkyl ethers with high-atom economy and low waste production in comparison to traditional routes involving protection/deprotection steps. The amphiphilic properties of these ethers (but also the acetal intermediates) were measured and a C12 alkyl chain has the optimum length to decrease the surface tension of water $\left(\mathrm{CMC}=0.012 \mathrm{mM}, \gamma \mathrm{sat} .=30 \mathrm{mN} . \mathrm{m}^{-1}\right)$. The antimicrobial activities were also evaluated on Gram-negative and Gram-positive bacteria. No activity was found against Gramnegative bacteria (MIC $>4 \mathrm{mM}$ ). On the contrary, interesting antimicrobial activities (MIC $\geq 0.02 \mathrm{mM}$ ) were obtained on Gram-positive bacteria such as L. monocytogenes, E. faecalis, E. faecium and S. aureus for methyl glycopyranosides bearing a C12 alkyl chain. More importantly, some of these 
compounds are also active against antibiotic-resistant bacteria which represent a major public health problem. These compounds have a great potential to be used as additives in pharmaceutical and/or cosmetic formulations and can find applications in food industry, in hospital and in other nonmedical fields.

\section{Experimental Section}

\section{General procedure A: acetalisation of methyl pyranosides}

In a dry two-necked round bottom flask, the corresponding methyl glycopyranoside (2.0 equiv) and sodium sulfate (1.5 equiv) were added in dry THF under argon. Dodecanal (1.0 equiv) was added portionwise over a 1-min period, followed by Amberlyst 15 (20wt\%/aldehyde). The mixture was stirred at $66^{\circ} \mathrm{C}$ for the time stated. After cooling down to room temperature, the reaction mixture was filtered, washed with $\mathrm{CH}_{2} \mathrm{Cl}_{2}(2 \times 25 \mathrm{~mL})$ and the filtrate was concentrated under reduced pressure. The residue was purified by flash chromatography (Cyclohexane : EtOAc) to give the corresponding methyl pyranoside acetals $6, \mathbf{1 2}-15$. See supporting information for characterization details.

\section{General procedure B: reductive cleavage of methyl pyranoside acetals}

In a 100-mL stainless steel autoclave, the corresponding methyl dodecylidene pyranoside (1.0 equiv) was dissolved in dry CPME (cyclopentyl methyl ether) and 5\%-Pd/C (5 mol\% in Pd) was added. The reactor was tightly closed, purged three times with hydrogen and pressurized at 40 Bar. The solution was then heated at $130^{\circ} \mathrm{C}$ for 16 hours. After cooling to room temperature, hydrogen pressure was released and the reaction mixture was dissolved in absolute $\mathrm{EtOH}(100 \mathrm{~mL})$ and filtered (Millipore Durapore filter $0.01 \mu \mathrm{m}$ ). The filtrate was evaporated under reduced pressure and the residue was purified by flash chromatography (Cyclohexane : EtOAc) to give the corresponding methyl pyranoside ethers 11, 16-18. See supporting information for characterization details.

\section{Physico-chemical studies}

The surface tensions were measured at $(25.0 \pm 0.1)^{\circ} \mathrm{C}$ with a K100MK2 Krüss tensiometer using a platinum rod as the probe, allowing to carry out experiments in small vessels. A total of $2.5 \mathrm{~mL}$ of water was first introduced in the vat. The solution was gradually concentrated with a stock surfactant solution (a manual dilution keeping volume constant was performed) and for each concentration the surface tension was measured until a stable value was obtained (standard deviation of the 5 final values of $0.2 \mathrm{mN} \cdot \mathrm{m}^{-1}$ ). 


\section{Antimicrobial assays}

The minimal inhibitory concentration experiments were realized on Gram-positive strain in accordance with the Clinical-Laboratory-Standards-Institute $\left(6^{\text {th }}\right.$ ed. Approved standard M100-S17. CLSI, Wayne, PA, 2007) recommendations.

\section{Acknowledgements}

The authors would like to thank Jesús Fermin Ontiveros for his contribution to the physico-chemical analyses. We also acknowledge the FINOVI foundation for financial support (AO9-07) to D.B. Finally we thank the company TEREOS and the Association Nationale de la Recherche et de la Technologie (ANRT) for their financial support through a CIFRE grant (2011/1660) to C.G.

[1] Antimicrobial agents, (Ed.: V. Bobbarala), InTech, Rijeka Croatia, 2012.

[2] A. Lucera, C. Costa, A. Conte, M. A. Del Nobile, Front. Microbiol. 2012, 3, 1-13.

[3] a) P. Suppakul, J. Miltz, K. Sonneveld, S. W. Bigger, J. Food Sci. 2003, 68, 408-420; b) S. Quintavalla, L. Vicini, Meat Sci. 2002, 62, 373-380; c) P. Appendini, J. H. Hotchkiss, Innovative Food Sci. Emerging Technol. 2002, 3, 113-126; d) S.-Y. Sung, L. T. Sin, T.-T. Tee, S.-T. Bee, A. R. Rahmat, W. A. W. A. Rahman, A.-C. Tan, M. Vikhraman, Trends Food Sci. Technol. 2013, 33, 110-123.

[4] a) Y. Gao, R. Cranston, Text. Res. J. 2008, 78, 60-72; b) B. Simoncic, B. Tomsic, Text. Res. J. 2010, 80, 1721-1737.

[5] a) Review on antimicrobial resistance, J. O'Neil, May 2016; b) Antimicrobial resistance global report on surveillance, World Health Organisation, 2014; c) H. C. Neu, Science 1992, 257, 1064-1072; d) T. F. Landers, B. Cohen, T. E. Wittum, E. L. Larson, Public Health Rep. 2012, 3, 1-13.

[6] J. S. Bradley, R. Guidos, S. Baragona, J. G. Bartlett, E. Rubinstein, G. G. Zhanel, M. D. Tino, D. L. Pompliano, F. Tally, P. Tipirneni, G. S. Tillotson, J. H. Powers, G. S. Tillotson, Lancet Infect. Dis. 2007, 7, 68-78.

[7] a) K. M. G. O'Connell, J. T. Hodgkinson, H. F. Sore, M. Welch, G. P. C. Salmond, D. R. Spring, Angew. Chem., Int. Ed. 2013, 52, 10706-10733; b) M. Basetti, M. Merelli, C. Temperoni, A. Astilean, Ann. Clin. Microbiol. Antimicrob. 2013, 12-22; c) C. T. Walsh, T. A. Wencewicz, J. Antibiot. 2014, 67, 7-22.

[8] a) E. Freese, C. W. Sheu, E. Galliers, Nature 1973, 241, 321-325; b) E. G. A. Verhaegh, D. L. Marshall, D.-H. Oh, Int. J. Food Microbiol. 1996, 29, 403-410. 

1029-1034.

[12] M. Ferrer, J. Soliveri, F. J. Plou, N. Lopez-Cortes, D. Reyes-Duarte, M. Christensen, J. L. Copa-Patino, A. Ballesteros, Enzyme Microb. Technol. 2005, 36, 391-398.

[13] a) A. Smith, P. Nobmann, G. Henehan, P. Bourke, J. Dunne, Carbohydr. Res. 2008, 343, 2557-2566; b) P. Nobmann, P. Bourke, J. Dunne, G. Henehan, J. Appl. Microbiol. 2010, 108, 2152-2661.

[14] P. Nobmann, A. Smith, J. Dunne, G. Henehan, P. Bourke, Int. J. Food Microbiol. 2009, 128, 440-445.

[15] a) M. le Maire, P. Champeil, J. V. Møller, Biochim. Biophys. Acta. 2000, 1508, 86-111; b) J. Piao, S. Kishi, S. Adachi, Colloids Surf. A Physicochem. Eng. Aspects 2006, 277, 15-19.

[16] a) M. N. Jones, Int. J. Pharm. 1999, 177, 137-159; b) H. Ahyayauch, M. Bennouna, A. Alonso, F. M. Goñi, Langmuir 2010, 26, 7307-7313.

[17] A. Ruzin, R. P. Novick, J. Bacteriol. 2000, 182, 2668-2671.

[18] H. S. Ved, E. Gustow, V. Mahadevan, A. Pieringer, J. Biol. Chem. 1984, 259, 8115-8121.

[19] R. Miethchen, I. Holz, H. Prade, A. Liptak, Tetrahedron 1992, 48, 3061-3068

[20] a) C. Gozlan, R. Lafon, N. Duguet, A. Redl, M. Lemaire, RSC Adv. 2014, 4, 50653-50661; b) C. Gozlan, E. Deruer, M.C. Duclos, V. Molinier, J.-M. Aubry, A. Redl, N. Duguet, M. Lemaire, Green Chem. 2016, 18, 1994-2004.

[21] These results were first patented: a) C. Gozlan, N. Duguet, M. Lemaire, M.-C. Duclos, O. Dumitrescu, G. Lina, A. Redl, Fr. Demande 2016, FR 3030279; b) C. Gozlan, D. Belmessieri, M.-C. Duclos, N. Duguet, M. Lemaire, G. Lina, O. Dumitrescu, A. Redl, PCT Int. Appl. 2016, WO 2016098046.

[22] See supporting information for calculations of green metrics.

[23] P. T. Anastas, J. C. Warner, Green Chemistry: Theory and Practice, Oxford University Press, New York, 1998.

[24] V. Molinier, J.-M. Aubry, Carbohydr. Chem. 2014, 40, 51-72.

[25] A. Cornella, L. Perez, F. Comelles, I. Ribosa, A. Manresa, M. T. Garcia, J. Colloid Interf. Sci. 2011, 355, 164-171.

[26] a) G. Rauwel, L. Leclercq, J. Criquelion, J.-M. Aubry, V. Nardello-Rataj, J. Colloid Interface Sci. 2012, 374, 176-186; b) D. N. Rubingh, Mixed micelle solutions, in: K.L. Mittal (Ed.), Solution Chemistry of Surfactants, Plenum, New York, 1979, pp. 337-354.

[27] L. Vanbaelinghem, P. Godé, G. Goethals, P. Martin, G. Ronco, P. Villa, Carbohydr. Res. 1998, 311, 89-94.

[28] M. F. K. Ariffin, M. Suffian, M. Annuar, T. Heidelberg, J. Surfactants Deterg. 2014, 17, 683-692.

[29] M. Ferrer, F. Comelles, F. J. Plou, M. A. Cruces, G. Fuentes, J. L. Parra, A. Ballestreros, Langmuir 2002, 18, 667-673.

[30] K. Shinoda, T. Yamaguchi, R. Hori, Bull. Chem. Soc. Jpn. 1961, 34, 237-241. 
[32] Minimum Inhibitory Concentration (MIC) values are defined as the lowest concentration of compound that shows no cell growth for all the replicates compared to the negative control after $18 \mathrm{~h}$.

[33] See supporting information for experimental details and the MIC values obtained against gram-negative strains.

[34] The Minimum Bactericidal Concentration (MBC) was determined by diluting the well below the MIC. The enumeration was then carried out after plating for $18 \mathrm{~h}$. The MBC corresponds to the lowest concentration of antimicrobial agent required to decrease the initial bacterial inoculum by at least $99.9 \%$.

[34] S. Schereier, S. V. P. Malheiros, E. De Paula, Biochimi. Biophys. Acta, 2000, 1508, 210-234.

[35] See supporting information for experimental details.

[36] R. García-Muelas, N. López, J. Phys. Chem. C. 2014, 118, 17531-17537.

[37] a) A. S. Bayer, T. Schneider, H.-G. Sahl, Ann. N. Y. Acad. Sci. 2013, 1277, 139-158; b) B. A. Cunha, F. M. Pherez, Eur. J. Clin. Microbiol. Infect. Dis. 2009, 28, 831-833; c) T. Jones, M. R. Yeaman, G. Sakoulas, S.-J. Yang, R. A. Proctor, H.-G. Sajl, J. Schrenzel, Y.-Q. Xiong, A. S. Bayer, Antimicrob. Agents Chemother. 2008, 52, 269-278; d) M. K. Hayden, K. Rezai, R. A. Hayes, K. Lolans, J. P. Quinn, R. A. Weinstein, J. Clin. Microbiol. 2005, 43, 5285-5287; e) A. Mangili, I. Bica, D. R. Snydman, D. H. Hamera, Clin. Infect. Dis. 2005, 40, 1058-1060.

[38] N. W. H. Cheetham, S. Padmini, J. Chromarogr. 1981, 208,100-103.

[39] P. Carcabal, R. A. Jockusch, I. Hünig, L. C. Snoek, R. T. Kroemer, B. G. Davis, D. P. Gamblin, I. Compagnon, J. Oomens, J. P. Simons, J. Am. Chem. Soc. 2005, 127, 11414-11420.

[40] V. Kräutler, M. Müller, P. Hünenberger, Carbohydr. Res. 2007, 342, 2097-2124.

[41] P. Angibeaud, J.-P. Utille, J. Chem. Soc., Perkin Trans. 1, 1990, 1490-1492. 